Research Paper:

\title{
A Simplified Approach to Evaluate Retinal Blue Light Hazard Using the Correlated Color Temperature of LED Light Sources
}

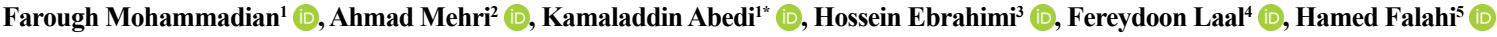

1. Department of Occupational Health and Safety Engineering, Environmental Health Research Center, Research Institute for Health Development, Kurdistan University of Medical Sciences, Sanandaj, Iran.

2. Department of Occupational Hygiene, School of Public Health, Hamadan University of Medical Sciences, Hamadan, Iran.

3. Department of Occupational Health and Safety Engineering, Shcool of Industry, Iran University of Medical Sciences, Tehran, Iran.

4. Department of Occupational Health, Social Determinants of Health Research Center, School of Health, Birjand University of Medical Sciences, Birjand, Iran

5. Department of Safety Engineering, Health and Environment, Faculty of Industry, Qazvin Branch, Islamic Azad University, Qazvin, Iran.

\begin{tabular}{|l|l|}
$\begin{array}{c}\text { Use your device to scan } \\
\text { and read the article online }\end{array}$ & $\begin{array}{l}\text { Cittation Mohammadian F, Mehri A, Ebrahimi H, Laal F, Falahi H, Abedi K. A Simplified Approach to Evaluate Retinal Blue } \\
\text { Light Hazard Using the Correlated Color Temperature of LED Light Sources. Journal of Advances in Environmental Health } \\
\text { Research. 2021; 9(4):299-310. http://dx.doi.org/10.32598/JAEHR.9.4.1226 }\end{array}$ \\
dol: $:$ http://dx.doi.org/10.32598/JAEHR.9.4.1226
\end{tabular}

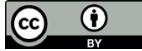

Article info:

Received: 10 Apr 2021

Accepted: 23 Aug 2021

Publish: 01 Oct 2021

\section{Keywords:}

Blue light hazard, Permissible exposure time, Light-Emitting Diode (LED), Correlated color temperature

\section{ABSTRACT}

Background: Visible light of short wavelength (blue light) can damage the retina cells, called blue light hazard. To protect the retina, permissible exposure limits have been determined by ICNIRP (the International Commission on Non-Ionizing Radiation Protection) and other health and safety agencies. These limits include complex physical concepts, and their evaluation requires light measuring instruments equipped with a blue light hazard function filter, which is not available at the moment in developing countries. This study aims to assess retinal blue light hazard using the correlated color temperature of Light-Emitting Diode (LED) light sources.

Methods: In this study, a new method was proposed for evaluating the retinal blue light hazard using only the Correlated Color Temperature (CCL) of LED light sources for which concern on blue light hazard has currently been reinforced. In the proposed method, the radiometric quantity (blue light effective radiance) in $\mathrm{W} \cdot \mathrm{m}^{-2} . \mathrm{sr}^{-1}$ was converted to the corresponding photometric quantity (blue light effective luminance) in $1 \mathrm{~m}^{\mathrm{m}} \mathrm{m}^{-2} \cdot \mathrm{sr}^{-1}$. Then a computer code was developed for relevant calculations.

Results: The estimated permissible exposure times applying the new photometry approach were highly reliable and supported by the literature. The proposed method is particularly useful because it eliminates the need to utilize any light measuring device.

Conclusion: The new approach makes the concept of blue light hazard straightforward and understandable for all specialists dealing with optical radiation safety.

\section{* Corresponding Author:}

Kamaladdin Abedi, PhD.

Address: Department of Occupational Health and Safety Engineering, Environmental Health Research Center, Research Institute for Health Development, Kurdistan University of Medical Sciences, Sanandaj, Iran.

Phone: +98 (87) 3364660

E-mail: kamal.abedi@gmail.com 


\section{Introduction}

I ntense visible radiation may cause a photochemical injury in the human eye called "photoretinitis" or "blue-light retinal injury" [1]. The potential for this damage resulting from exposure of the eye to the highest energy wavelengths of the visible spectrum (between 400-700 nanometre (nm) is called "Blue Light Hazard" (BLH) $[2,3]$. The blue light can penetrate deeply into the eye, reach the retina and cause phototoxic effects in the retina cells. Despite the higher energy of Ultraviolet (UV), it does not pass through the cornea and lens and reach the retina (except for the sub-area of 300-400 nm for which about $1 \%-2 \%$ of the incoming UV can reach the retina) $[1,4]$. Therefore, the most important electromagnetic spectrum responsible for BLH falls into the range from $300 \mathrm{~nm}$ (UV) to $700 \mathrm{~nm}$ (red), with the sensitivity peak around $440 \mathrm{~nm}$ (blue) [5].

Light-induced retinal impairment can be attributed to at least three different mechanisms: mechanical, thermal, and photochemical [6]. The former is caused by shock waves at severe light intensities for short exposure times. The second is caused when a retinal temperature shift of $10^{\circ} \mathrm{C}-20^{\circ} \mathrm{C}$ is achieved after short-term exposure to high-power density light. The latter, which is the mechanism responsible for BLH results when the individual observes high-energy short wavelengths for a long time period $[4,7,8]$. Previous studies have shown that the BLH mechanism will be dominated when the exposure time exceeds 10 seconds (the observer looks at the direct blue light for a long time). The symptoms can be seen as blind spots or vision loss at some points on the retina, although some recovery will be achieved over time [9-11].

One of the main light sources for which concerns over BLH have intensified, is a Light-Emitting Diode (LED) $[12,13]$. Contrary to the higher radiant power emission of incandescent lamps, LEDs produce more light thanks to the smaller emission angles and much higher luminous efficacy. Moreover, due to a strong emission peak near $440 \mathrm{~nm}$ with blue and even multi-color and white LEDs, the BLH risk for LED luminaires is believed to be several times higher than other light sources $[1,12]$.

According to ACGIH (The American Conference of Governmental Industrial Hygienists) threshold limit values (TLVs), the time-integrated radiance weighted by BLH spectral function should be below the limit of 100 $\mathrm{J} . \mathrm{cm}^{-2} \mathrm{sr}^{-1}$ for an exposure time of 167 minutes during a day. For exposure durations longer than 167 minutes, radiance weighted by BLH spectral function should not exceed $10 \mathrm{~mW} \cdot \mathrm{cm}^{-2} \mathrm{sr}^{-1}[14,15]$. Assessing and characterizing whether the TLVs are exceeded or not requires a broadband detector equipped with a BLH spectral function filter which is not available at the moment in developing countries, and if made, it will be costly [4]. Therefore, finding new approaches for exposure assessment without using broadband and filtered detectors will be appreciated, especially by professionals dealing with optical radiation safety. This study attempts to propose a novel simplified method to assess BLH of LED light sources using the Correlated Color Temperature (CCT) of the lamps, replacing photometry with radiometry. The luminaire colorimetric properties can be a convenient and simple way to estimate the BLH without employing any radiometer.

This study attempts to propose a novel simplified method to evaluate BLH of LED light sources using the CCT of the lamps, replacing photometry with radiometry. Using the luminaire colorimetric properties can be a convenient and simple way to evaluate the BLH without employing any radiometer.

\section{Materials and Methods}

The output intensities of all visible light sources, including Light-Emitting Diodes (LEDs), can be specified in two different quantities: radiometric and photometric, which are expressed as radiant intensity and luminous intensity, respectively. The radiant flux (also called radiance) is measured in $\mathrm{W} \cdot \mathrm{m}^{-2} \cdot \mathrm{sr}^{-1}$, and the luminous flux (also called luminance) is measured in $\mathrm{cd}^{-\mathrm{m}^{-2}}$ or nit Since the photometry units take the human eye physiology and color perception into account, the visible light intensity is mostly given in photometry units. The radiometric values can simply be converted to photometric values through the Equation $1[16,17]$ :

$$
\text { 1. } \varphi v=K_{m} \int_{380}^{760} L(\lambda, I) v(\lambda) d \lambda
$$

In this Equation, $\varphi v$ is the luminance (photometric value) in $1 \mathrm{~m} \cdot \mathrm{m}^{-2} \cdot \mathrm{sr}^{-1}$ or nit, $\mathrm{Km}=683 \mathrm{~lm} \cdot \mathrm{W}^{-1}$ is the maximum value of the spectral luminous efficacy, $\mathrm{L}_{(\lambda, \mathrm{T})}$ is the spectral radiance in $\mathrm{W} \cdot \mathrm{m}^{-2} \cdot \mathrm{sr}^{-1} \cdot \mathrm{nm}^{-1}$, and $\mathrm{v}_{(\lambda)}$ refers to the photopic response of the human eye.

When the source resembles an ideal black body and emits light through thermal radiation (e.g., incandescent lamps), $\mathrm{T}$ is both the surface temperature and Color Temperature (CT) of the radiator [18]. The CT of a light source refers to the temperature of an ideal black body radiator that emits light with the same color as the light source does [19]. It is generally known that when an ob- 
ject is heated, it begins to glow; initially red, then as the temperature increases, brighter red, then bright orange, next brilliant white, and finally bluish white. The resulting luminance may be different from one material to another, but the color of the glow is essentially the same for all materials, and varies only with the temperature [19]. In the case where the radiator is an ideal black body, the spectral radiance $\left(\mathrm{L}_{(\lambda, \mathrm{T})}\right)$ can be described by Planck's Law (Equation 2) [20]:

$$
\text { 2. } \left.L_{(\lambda, T}\right)=\left(\frac{2 c^{2} h}{\lambda^{5}}\right)\left(e^{h c / k_{B} \lambda T}-1\right)^{-1}
$$

In this Equation, $\mathrm{L}_{(\lambda, \mathrm{T})}$ is the spectral radiance per unit wavelength in W.m ${ }^{-2} \cdot \mathrm{sr}^{-1} \cdot \mathrm{nm}^{-1}, \lambda$ is the wavelength in $\mathrm{nm}, \mathrm{T}$ is the $\mathrm{CT}$ in $\mathrm{K}, \mathrm{h}=6.626 \times 10^{-34}$ refers to the Planck's constant in J.s, $\mathrm{kB}=1.381 \times 10^{-23}$ is the Stephen Boltzmann's constant in J.K. $\mathrm{K}^{-1}$, and $\mathrm{c}=3.0 \times 108$ is the speed of light in $\mathrm{m} \cdot \mathrm{s}^{-1}$.

For the light sources that are imperfect black body radiators, the $\mathrm{CT}$ tends to be different from the actual temperature of the surface. Although with some approximations, incandescent lamps perform like an ideal black body, other light sources like fluorescent lamps and LEDs do not follow the Planck's spectral distribution, and CT cannot be assigned to them because, in these sources, the light is essentially generated through phenomena other than thermal radiation [21]. These light sources are labeled instead with CCT, which is based on the CT concept and derived from the ideal black body radiation. Like CT, CCT is also measured in kelvin degrees. Still, with some discrepancy compared to CT, it is defined as the color temperature of a reference source (black body radiator) which resembles the color of a given artificial light source from the viewpoint of human color perception [22]. Thus, for a non-ideal black-body source (e.g., a white LED) having a specified CCT, the luminance and radiance could be obtained by substituting CCT for T in Equations 1 and 2 [23].

\section{Blue light effective spectral radiance}

Since the BLH is limited to the wavelength range of 300-700 nm, the blue light effective spectral radiance $\left(\mathrm{L}_{\mathrm{B}}\right)$ of the LED source should be determined over this truncated spectrum. This quantity is obtained from integrating spectral radiance weighted against blue-light spectral hazard function and defined by ICNIRP (the International Commission on Non-Ionizing Radiation Protection) according to Equation 3 [10]:

$$
\text { 3. } \mathrm{L}_{\mathrm{B}}=\int_{300}^{700} L_{(\lambda, T)} B_{(\lambda)} d \lambda
$$

In this Equation, $\mathrm{B}_{(\lambda)}$ is the BLH weighting factor determined by ICNIRP as a function of wavelength. The $B_{(\lambda)}$ values, in the wavelength range of $300-700 \mathrm{~nm}$, are shown in Figure 1.

Converting the blue light effective spectral radiance to blue light effective spectral luminance

This study describes a new method for measuring BLH without using any radiometer and only by employing the CCT of a given light source. Since CCT is perceived through the eyes, it can only be used with photometric quantities. Therefore, the blue light effective spectral radiance, $L_{B}$, should be converted to its photometric equivalent (namely here the blue light effective spectral luminance), $\varphi_{B}$, in the wavelength region of 300-700 $\mathrm{nm}$, which is the region responsible for BLH according to ICNIRP exposure limit values [10].

To convert the radiant flux to its equivalent luminous flux for any source with any spectral radiant power distribution is simply a matter of integration, as Equation 1 represents. In the case where the blue light effective spectral luminance is needed to be determined, another factor, $\mathrm{B}_{(\lambda)}$ (from Equation 3), also appears in the integration besides $\mathrm{V}_{(\lambda)}$, and the chosen wavelength range should be that results in BLH (300-700 nm). But, the lower limit of integral could not be $300 \mathrm{~nm}$ for photometric quantities because the wavelength range of $\mathrm{V}$ is 380 to $700 \mathrm{~nm}$. Therefore, the lower limit of integration can be changed from 300 to $380 \mathrm{~nm}$, which influences the effective spectral luminance insignificantly, and the resultant error is negligible, as described in the following. Finally, the blue light effective spectral luminance, $\varphi_{\mathrm{B} \text { (calculated) }}$, is yielded according to Equation 4:

$$
\text { 4. } \varphi_{B(\text { calculated })}=683\left\{\int_{380}^{700}\left[\left(\frac{2 c^{2} h}{\lambda^{5}}\right)\left(e^{h c / k_{B}^{\lambda T}}-1\right)^{-1} B(\lambda) v_{(\lambda)} d\right]\right\}
$$

The values of $\mathrm{B}_{(\lambda)}$ and $\mathrm{v}_{(\lambda)}$ vary with wavelength, and their amounts are listed in Table 1 for a wavelength range of 380-700 $\mathrm{nm}$.

The new parameter of $\gamma_{(\lambda)}$

In Equation 4, the multiple of $\mathrm{B}_{(\lambda)}$ and $\mathrm{V}_{(\lambda)}$ can be defined as a new factor, i.e., $\gamma_{(\lambda)}$, which represents the BLH considering photopic eye sensitivity. Figure 2 illustrates $\gamma_{(\lambda)}$ in addition to both $\mathrm{B}_{(\lambda)}$ and $\mathrm{V}_{(\lambda)}$ curves. As seen in Figure 2, the maximum amount of $\gamma_{(\lambda)}$ curve, which is the resultant of BLH function times eye sensitivity function, is located around $480 \mathrm{~nm}$ wavelength with the peak value of 0.07 . For assessing BLH by our proposed photometry method, the new parameter of $\gamma_{(\lambda)}$ should be taken into consider- 


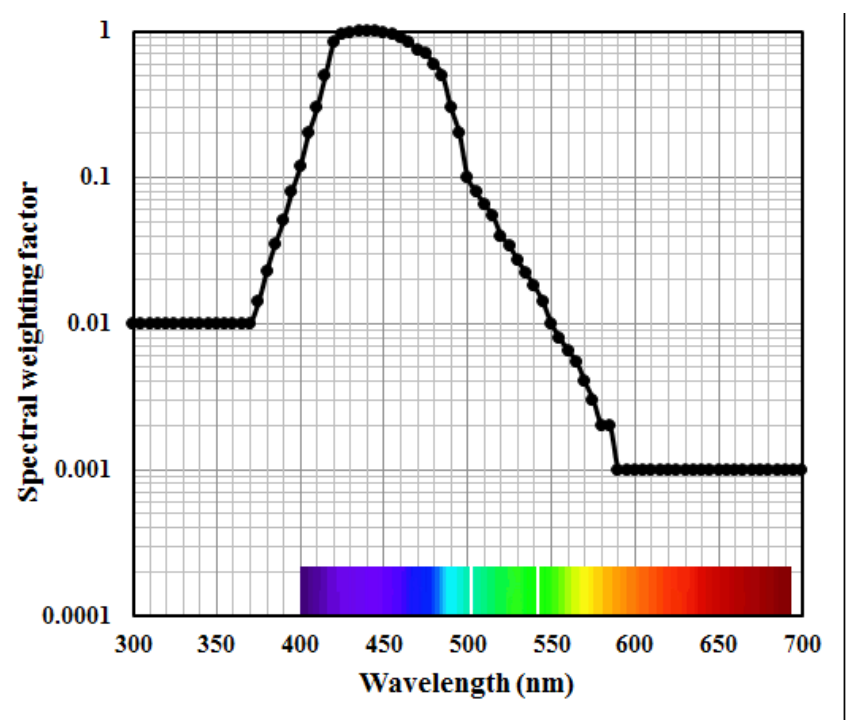

Figure 1. Blue light hazard weighting factor $\left(\mathrm{B}_{(\lambda)}\right)$ as a function of wavelength

ation, and then Equation 4 can be rewritten according to Equation 5:

$$
\text { 5. } \varphi_{B(\text { calculated })}=683\left(\int_{(\lambda, \mathrm{T})} y_{(\lambda)} d \lambda\right)
$$

In this Equation, $\gamma_{(\lambda)}$ is the defined new parameter used in the photometry method. As represented in Figure 2, $\gamma_{(\lambda)}$ has very low values in the sub-area of 300 to $380 \mathrm{~nm}$, leading to a very low amount of $\varphi_{\mathrm{B} \text { (calculated) }}$ in this region, which can be considered negligible. Therefore, excluding this region from calculations had no significant impact on the final yields of $\varphi_{\mathrm{B} \text { (calculated) }}$. However, ignoring part of the spectrum can cause errors in results, and estimating the error values is necessary. To this end, the net contribution of part of the spectrum with no photopic sensitivity (300-380 $\mathrm{nm}$ ) should be determined on the effective spectral radiance calculations. In other words, the ratio of the area between 305 to $380 \mathrm{~nm}$ to the total area between 305 to $700 \mathrm{~nm}$ must be specified, as depicted in Figure 3. This ratio and subsequent related calculations allow us to realize whether excluding 305-380 $\mathrm{nm}$ from computations is considerable or not.

\section{Conversion of radiometric exposure limits to photometric exposure limits}

The ICNIRP exposure limit for protecting the retina from photochemical injury for an apparent diameter of the light source greater than $0.011 \mathrm{rad}$ [5], and for exposure duration less than $10000 \mathrm{~s}$, has been determined as according to Equation 6 [10]:

$$
\text { 6. } L_{B}=\int_{(\lambda, \mathrm{T})} B_{\lambda} d \lambda \leq\left(10^{6} \mathrm{Jm}^{-2} \cdot s r^{-1}\right) / t(s)
$$

By ignoring the $300-380 \mathrm{~nm}$ spectrum and with a minor error, Equation 6 could be rewritten as according to Equation 7:

$$
\text { 7. } L_{B}=\int L_{(\lambda, \mathrm{T})} B_{\lambda} d \lambda \approx L_{B(\text { photopic region })}=\int L_{(\lambda, \mathrm{T})} B_{\lambda} d \lambda \leq
$$

$$
\left(10^{6} \mathrm{Jm}^{-2} . s r^{-1}\right) / t(s)
$$

As Equation 7 represents, LB(Photopic region) should not exceed $106 \mathrm{~J} . \mathrm{m}^{-2} \cdot \mathrm{sr}^{-1}$ per unit of time. However, this radiometric quantity should be converted to its equivalent photometric quantity, $\varphi_{\mathrm{B} \text { (calculated) }}$, in Talbots. $\mathrm{m}^{-2} \cdot \mathrm{sr}^{-1}$ (lumen.s. $\mathrm{m}^{-2} \cdot \mathrm{sr}^{-1}$ ), in order to become appropriate and useful in our proposed method. Therefore, both sides of inequality in Equation 7 must be multiplied by the maximum luminous efficacy of $683 \mathrm{~lm} . \mathrm{W}^{-1}$, and also by the photopic response, $\mathrm{V}_{(\lambda)}$, in the wavelength region of 380$700 \mathrm{~nm}$ as described previously (Equation 8):

$$
\begin{gathered}
\text { 8. } \varphi_{B(\text { calculated })}=683\left(\int_{(\lambda, \mathrm{T})} y_{(\lambda)} d \lambda\right) \int_{(\lambda, \mathrm{T})} B_{\lambda} d \lambda=683\left(\int L\right. \\
{ }_{(\lambda, \mathrm{T})} B_{(\lambda)} v_{(\lambda)} d \lambda \leq\left(10^{6} 683 \int_{(\lambda)} d \lambda t(s)\right)
\end{gathered}
$$

Using Table 1 and considering $\Delta \lambda$ intervals to be $9 \mathrm{~nm}$, then the summation amount of photopic sensitivity of the human eye, S, for the entire specified range of wavelength can be according to Equations 9, 10, 11 and 12:

$$
\begin{aligned}
& \text { 9. } S=\int v_{(\lambda)} d \lambda \\
& \text { 10. } \varphi_{B(\text { calculated })}=683\left(\int_{(\lambda, T)} y_{(\lambda)} d \lambda\right] \times t(s) \leq 10^{6} \times 683 \times 68.78
\end{aligned}
$$

683 can be cancelled out from two sides of inequality: 
Table 1. Values of blue light hazard function, $B_{(\lambda)^{\prime}}$ and photopic sensitivity $v_{(\lambda)}$

\begin{tabular}{|c|c|c|c|c|c|c|c|c|c|c|c|}
\hline$\lambda_{(n m)}$ & $\mathbf{i}$ & $\mathbf{B}_{(\lambda)}$ & $\mathbf{v}_{(\lambda)}$ & $\lambda_{(n m)}$ & $\mathbf{i}$ & $\mathbf{B}_{(\lambda(i))}$ & $\mathbf{v}_{(\lambda(i))}$ & $\lambda_{(n m)}$ & $\mathbf{i}$ & $\mathbf{B}_{(\lambda(i))}$ & $\mathbf{v}_{(\lambda(i))}$ \\
\hline 380 & 1 & 0.01 & 0.000039 & 470 & 19 & 0.62 & 0.09098 & 560 & 37 & 0.006 & 0.995 \\
\hline 385 & 2 & 0.0125 & 0.000079 & 475 & 20 & 0.55 & 0.115 & 565 & 38 & 0.005 & 0.974 \\
\hline 390 & 3 & 0.025 & 0.00012 & 480 & 21 & 0.45 & 0.13902 & 570 & 39 & 0.004 & 0.953 \\
\hline 395 & 4 & 0.05 & 0.000258 & 485 & 22 & 0.4 & 0.17352 & 575 & 40 & 0.003 & 0.9115 \\
\hline 400 & 5 & 0.1 & 0.000396 & 490 & 23 & 0.22 & 0.20802 & 580 & 41 & 0.002 & 0.87 \\
\hline 405 & 6 & 0.2 & 0.000803 & 495 & 24 & 0.16 & 0.26551 & 585 & 42 & 0.002 & 0.8135 \\
\hline 410 & 7 & 0.4 & 0.00121 & 500 & 25 & 0.1 & 0.323 & 590 & 43 & 0.001 & 0.757 \\
\hline 415 & 8 & 0.8 & 0.002605 & 505 & 26 & 0.079 & 0.413 & 600 & 44 & 0.001 & 0.694 \\
\hline 420 & 9 & 0.9 & 0.004 & 510 & 27 & 0.063 & 0.503 & 610 & 45 & 0.001 & 0.631 \\
\hline 425 & 10 & 0.95 & 0.0078 & 515 & 28 & 0.05 & 0.6065 & 620 & 46 & 0.001 & 0.503 \\
\hline 430 & 11 & 0.98 & 0.0116 & 520 & 29 & 0.04 & 0.71 & 630 & 47 & 0.001 & 0.381 \\
\hline 435 & 12 & 1 & 0.0173 & 525 & 30 & 0.032 & 0.786 & 640 & 48 & 0.001 & 0.265 \\
\hline 440 & 13 & 1 & 0.023 & 530 & 31 & 0.025 & 0.862 & 650 & 49 & 0.001 & 0.175 \\
\hline 445 & 14 & 0.97 & 0.0305 & 535 & 32 & 0.02 & 0.908 & 660 & 50 & 0.001 & 0.107 \\
\hline 450 & 15 & 0.94 & 0.038 & 540 & 33 & 0.016 & 0.954 & 670 & 51 & 0.001 & 0.061 \\
\hline 455 & 16 & 0.9 & 0.049 & 545 & 34 & 0.013 & 0.9744 & 680 & 52 & 0.001 & 0.032 \\
\hline 460 & 17 & 0.8 & 0.06 & 550 & 35 & 0.01 & 0.9949 & 690 & 53 & 0.001 & 0.0082 \\
\hline 465 & 18 & 0.7 & 0.07549 & 555 & 36 & 0.008 & 1 & 700 & 54 & 0.001 & 0.0041 \\
\hline
\end{tabular}

11. $\left.\varphi_{B(\text { calculated })}=\int L_{(\lambda, \mathrm{T})} y_{(\lambda)} d \lambda\right]\left(l m \cdot m^{-2} \cdot s r^{-1}\right) \times t(s)$

$\leq 68.78 \times 10^{6}\left(\right.$ Talbot. $\left.\mathrm{m}^{-2} . s r^{-1}\right)$

$$
12 . t(s) \leq \frac{68.78 \times 10^{6}(\text { Talbot })}{\varphi_{B(\text { calculated })}(\text { lm })}
$$

The Threshold Limit Value (TLV) for blue light-weighted effective radiance (radiometry method) has been set $10^{6}$ $\left(\mathrm{J} . \mathrm{m}^{-2} \cdot \mathrm{sr}\right)$. For the photometry method, the non-round number $68.78 \times 10^{6}\left(\right.$ Talbot.m $\left.{ }^{-2} \cdot \mathrm{sr}^{-1}\right)$ was calculated as $\varphi_{\mathrm{B}(\mathrm{TLV})}$. The value was then compared to the measurements and computations reported by Okuno et al. for various light sources [4]. For example, for a $500 \mathrm{~W}$ xenon lamp with a permissible exposure time of $0.91 \mathrm{~s}$, the blue light TLV quantity is achieved according to Equations 13, 14 and 15: 13.t $(s)=\frac{10^{6}\left(J \cdot m^{-2} \cdot s r\right)}{\left.\int_{380}^{700} L_{(\lambda, T}\right) B_{(\lambda)} d \lambda}=\frac{\varphi_{B(T L V)}\left(\operatorname{lm} \cdot s \cdot m^{-2} \cdot s r\right)}{\left.\int_{380}^{700} \int_{(\lambda, P}\right) B_{(\lambda)} d \lambda}$

14. $0.91(\mathrm{~s})=\frac{10^{6}\left(\mathrm{~J} \mathrm{~m}^{-2} \mathrm{sr}\right)}{1100000\left(\mathrm{~W} \mathrm{~m} \mathrm{~m}^{-2} \mathrm{sr}\right)}=\frac{\left.\varphi_{B(\text { TLV })} \text { (lumen } \cdot \mathrm{s} \cdot \mathrm{m}^{-2} \cdot \mathrm{sr}\right)}{683 \times 110675\left(\text { lumen } \cdot \mathrm{s} \cdot \mathrm{m}^{-2} \cdot \mathrm{sr}\right)}$

15. $\varphi_{B(T L V)}=68787833$ (nit.s)

$\Phi_{\mathrm{B} \text { (calculated) }}$ should be easily computed for the proposed method to be applicable. Acceptable exposure duration for a LED light source with a specified CCT will then be determined corresponding to ICNIRP exposure limits for BLH. This event is conveniently made possible using an Originpro computing code. 


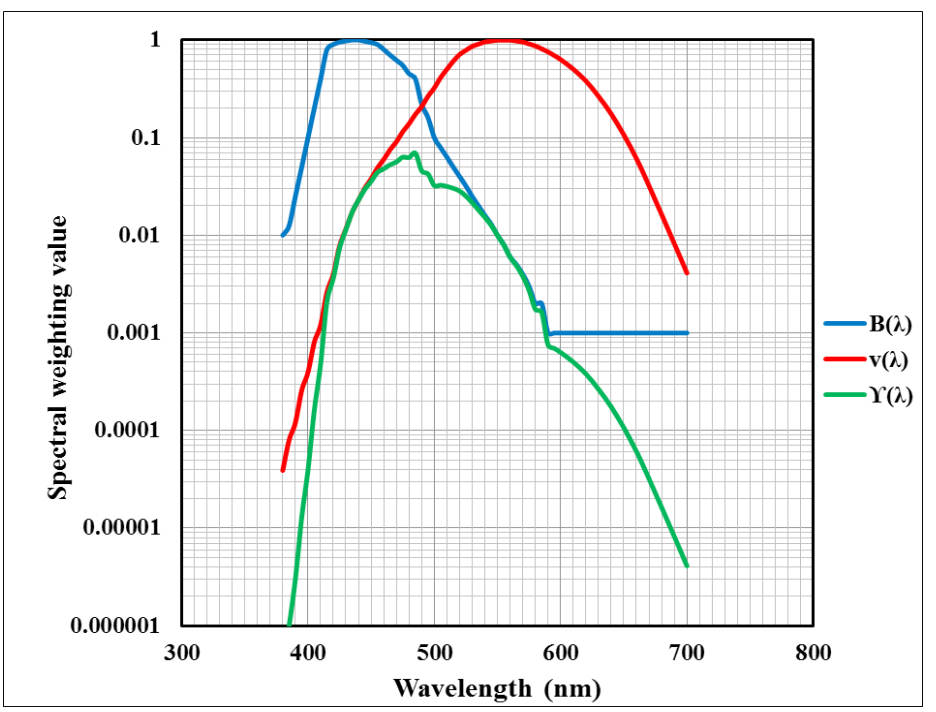

Figure 2. Blue light hazard function, $\mathrm{B}_{(\lambda)^{\prime}}$ Photopic function, $\mathrm{V}_{(\lambda)^{\prime}}$ and photopic blue light hazard function, $\gamma_{(\Lambda)^{\prime}}$ depicted for wavelength range of $380-700 \mathrm{~nm}$

\section{Results and Discussion}

The effect of excluding non-photopic region on results

According to ICNIRP guidelines, the wavelength range of concern for BLH is 300-700 nm [5]. As previously described, for our proposed photometric method of BLH measurement to be acceptable, the non-photopic wavelength range of 300-380 nm should be excluded from computations. Although this region is located within the UV spectrum, and due to the high UV absorbing power of the anterior structure of the human adult eyes, only $1 \%-2 \%$ of the incoming light belonging to this sub-area will reach the retina. However, truncating the wavelength band may always cause some errors in results, and determining the error rate is highly required. Therefore, it is needed to specify to what extent the spectral radiance is influenced by the energy coming from the non-photopic region $(300-380 \mathrm{~nm})$. In other words, the impact of the light energy belonging to the mentioned spectrum (the rectangular area between the Y-axis line and the dashed line indicating $380 \mathrm{~nm}$ in Figure 3) on the total radiance amounts needs to be identified. In this regard, the Originpro code was executed with and without the non-photopic range of wavelength for different temperatures. The results are shown in Table 2 indicating no significant difference between two different modes of

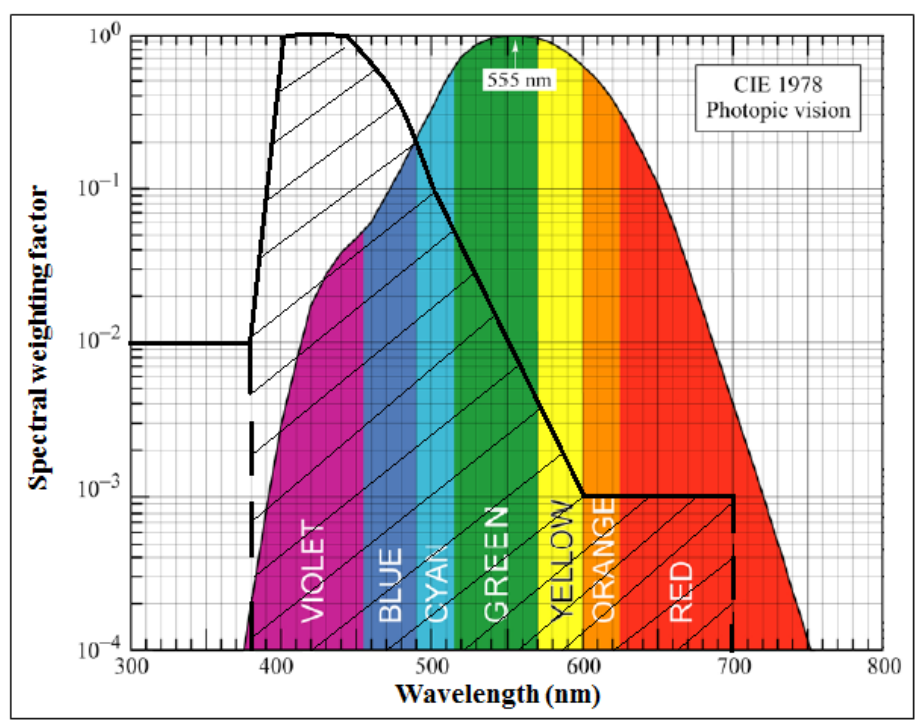

Figure 3. Part of the spectrum for blue light hazard function, $\mathrm{B}_{(\lambda)^{\prime}}$ having overlap with photopic function, $\mathrm{V}_{\left(\mathrm{x}^{\prime}\right.}$, compared to the part without overlap 
Table 2. The calculations of light energy with and without the non-photopic range of wavelength for different temperatures using originpro software

\begin{tabular}{|c|c|c|c|}
\hline $\begin{array}{l}\text { Correlated Color Temperature } \\
\text { (K) }\end{array}$ & $\begin{array}{c}\left.\int_{300}^{700} L_{(\lambda, P}\right) B_{(\lambda)} \Delta \lambda \\
\left(\mathbf{W} \cdot \mathbf{m}^{-\mathbf{2}} \mathbf{s r}^{-\mathbf{1}} \mathbf{)}\right.\end{array}$ & $\begin{array}{c}\left.\int_{300}^{700} L_{(\lambda, T}\right) B_{(\lambda)} \Delta \lambda \\
\left(\mathbf{W} \cdot \mathbf{m}^{-\mathbf{2}} \mathbf{s r}^{-\mathbf{1}} \mathbf{)}\right.\end{array}$ & Relative Error (\%) \\
\hline 2000 & $4.465994 \times 10^{6}$ & $4.467 \times 10^{6}$ & 0.03 \\
\hline 2200 & $1.8272320 \times 10^{7}$ & $1.8281625 \times 10^{7}$ & 0.05 \\
\hline 2400 & $5.9568 \times 10^{7}$ & $5.96112 \times 10^{7}$ & 0.07 \\
\hline 2500 & $1.003992 \times 10^{8}$ & $1.004843 \times 10^{8}$ & 0.08 \\
\hline 2700 & $2.547308 \times 10^{8}$ & $2.550157 \times 10^{8}$ & 0.11 \\
\hline 3000 & $8.199128 \times 10^{8}$ & $8.212115 \times 10^{8}$ & 0.15 \\
\hline 3500 & $3.715385 \times 10^{9}$ & $3.724626 \times 10^{9}$ & 0.24 \\
\hline 4000 & $1.160515 \times 10^{10}$ & $1.16458 \times 10^{10}$ & 0.35 \\
\hline 5000 & $5.76187 \times 10^{10}$ & $5.794704 \times 10^{10}$ & 0.56 \\
\hline 6000 & $1.685154 \times 10^{11}$ & $1.698513 \times 10^{11}$ & 0.78 \\
\hline
\end{tabular}

calculations, and the relative error was negligible in all cases (less than $1 \%$ ), particularly when the CCLs were lower than $3000 \mathrm{~K}$ (relative error $<0.2 \%$ ).

The negligible effect of the truncated area on results can be explained by several reasons. Firstly, it covers a tiny area (Figure 3 ), and considering $\mathrm{d} \lambda$ intervals to be $10 \mathrm{~nm}$ for integration, only 8 thin strips form it. Secondly, the value of $\mathrm{B}_{(\lambda)}$ receives very small amounts in this area (it is constant and equals $10^{-2}$ ), which results in very low yields of blue light effective radiance in this region. Hence, the specified band can be neglected in calculating either the total blue light effective radiance or luminance with an insignificant error. It should be noted here that ignoring the non-photopic region could be only acceptable for white light sources where the radiated light consists of the entire visible spectrum. For other narrow-band light sources like blue LEDs, it may not be allowable.

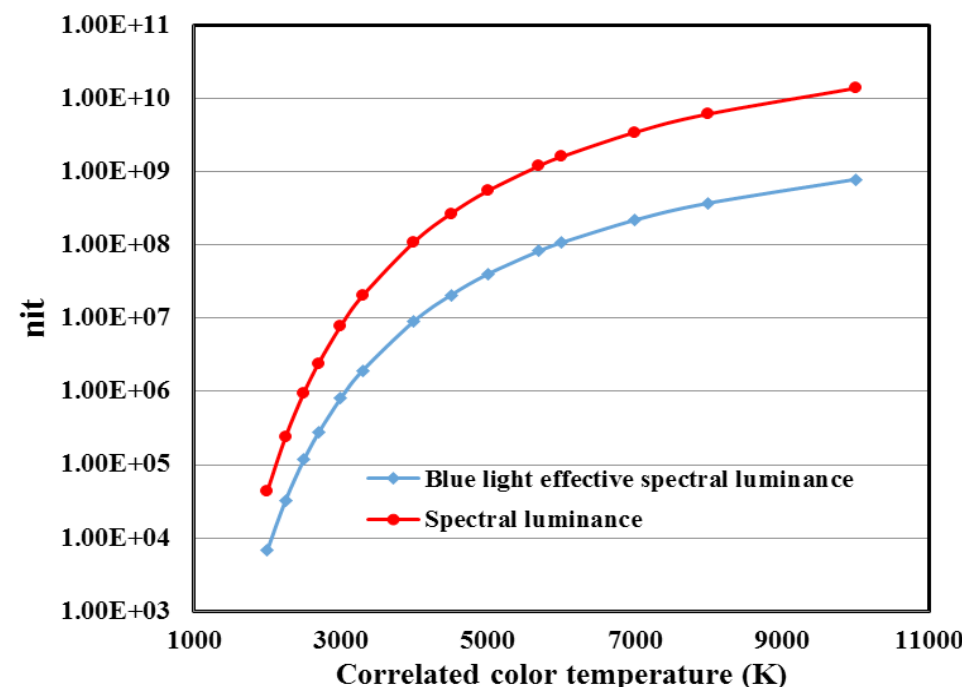

Figure 4. Calculated luminance and blue light effective luminance for the CCT range of 2000-10000 K using proposed photometry method 


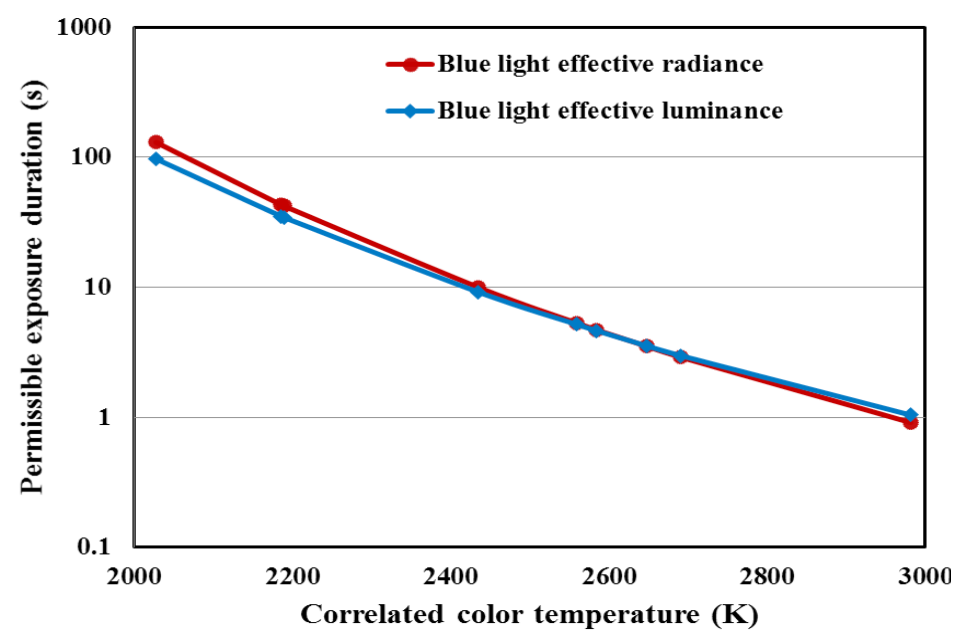

Figure 5. Permissible exposure duration estimated by both blue light effective radiance and blue light effective luminance as a function of CCT of the light source for a source with apparent diameter greater than $0.011 \mathrm{rad}$

Comparison of blue light effective radiance and blue light effective luminance

Comparing the obtained permissible exposure time based on the blue light effective radiance recommended by ICNIRP and blue light effective luminance proposed in this study enabled us to analyze the accuracy of our suggested photometry method. Firstly, the spectral luminance and blue light effective spectral luminance were compared to determine the effect of $V_{(\lambda)}$ and $\gamma_{(\lambda)}$ on the results. Figure 4 shows both the spectral luminance and blue light effective spectral luminance calculated by the proposed photometry method for the CCT range of 2000$10000 \mathrm{~K}$. Figure 4 shows that the trend is upward for both the spectral luminance and blue light effective spectral luminance as CCT increases, and input CCTs of 200010000 leads to a blue light effective luminance range of almost 104-109 $\mathrm{lm}$. However, for the same CCTs, the spectral luminance was assigned a range of about 105 $1010 \mathrm{~lm}$. For a lower specified CCT of $2000 \mathrm{~K}$, the blue light, effective spectral luminance was around $50000 \mathrm{~lm}$ smaller than spectral luminance, whereas, with higher CCTs, the discrepancy was much greater. This outcome means that although at higher CCTs, much cooler light is expected to be generated from light sources, which raises the level of blue light effective luminance, due to higher extents of $V_{(\lambda)}$ comparing $\gamma_{(\lambda)}$ for the whole spectrum, spectral luminance will always become a greater quantity. Secondly, the results of radiometry and photometry calculations were compared. Figure 5 represents the allowable exposure time estimated by blue light, effective radiance, and blue light effective luminance as a function of CCT of the light source. As Figure 5 shows, there are two nearly coincidental curves for the given CCT range, meaning that employing both calculation methods (radiometry and photometry) leads to the same results. There-
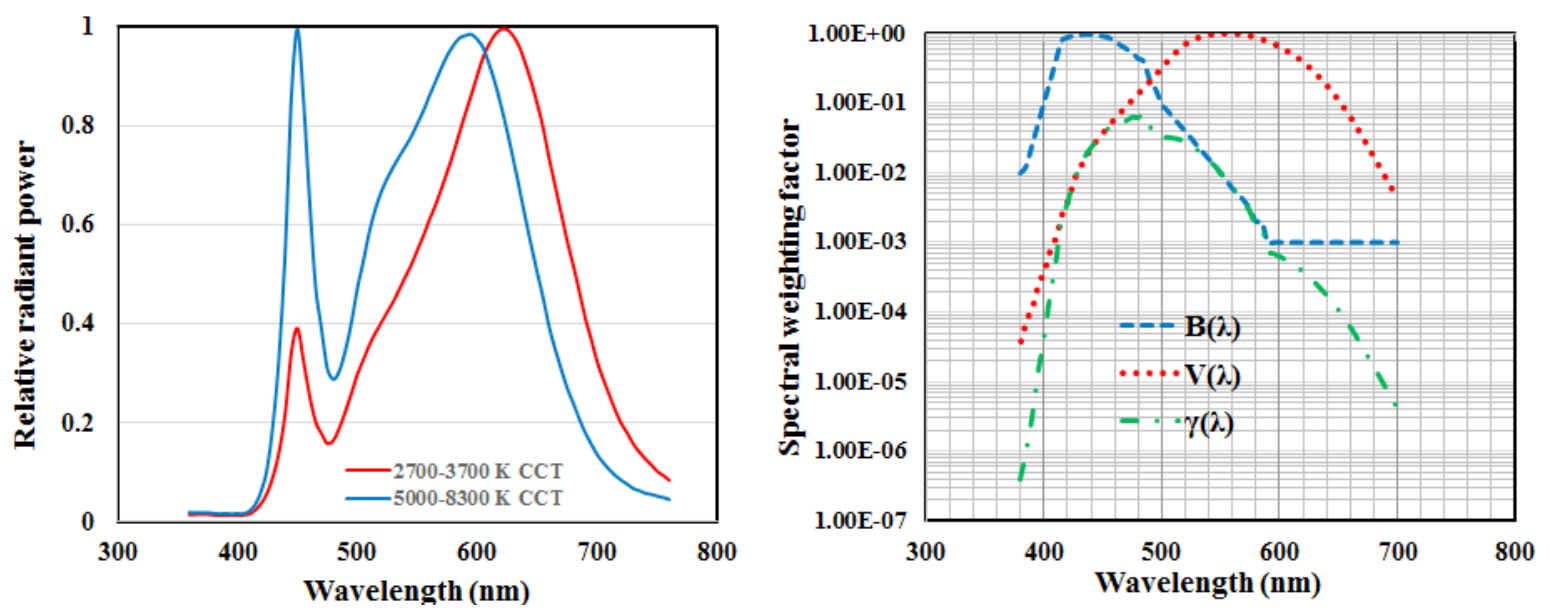

Figure 6. The spectral distribution of LEDs with different CCT range as compared to the spectral weighting functions of $\mathrm{B}_{(\lambda)^{\prime}}$ $\mathrm{V}_{(\lambda)^{\prime}}$ and $\gamma_{(\lambda)}$ 

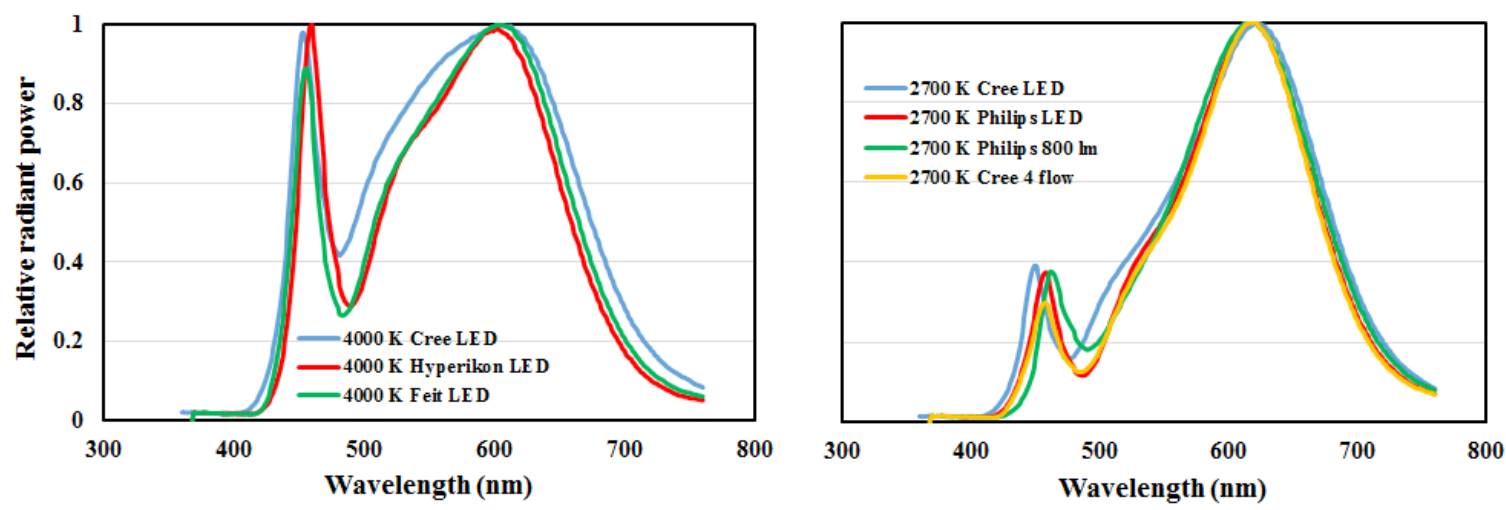

Figure 7. Comparing the spectral distribution of LEDs with the same CCT produced by different lighting companies

fore, the permissible exposure time estimated by the photometry method can be considered highly reliable. However, the findings of both curves (radiometry and photometry) in Figure 5 were obtained from calculations using Originpro 2017 software, and CCT of the light source was used as input data for both computations. No field measurement was carried out. Subsequently, uncertainty may arise on the accuracy of results. But the calculated values of the blue light effective radiance are fortunately supported by a few sets of data found in the literature. Okuno et al. carried out a study with very close and, in most cases, exactly similar findings for blue light effective radiance. They measured the effective radiance using a spectroradiometer and then weighted and integrated the data provided by the instrument against $\mathrm{B}_{(\lambda)}$ over the wavelength range of $380-780 \mathrm{~nm}$. Like our argumentation, they also noted that ignoring the wavelength range of 300-380 nm caused no significant error on the results "because the relative contribution of this range to effective radiance is negligible" [4].

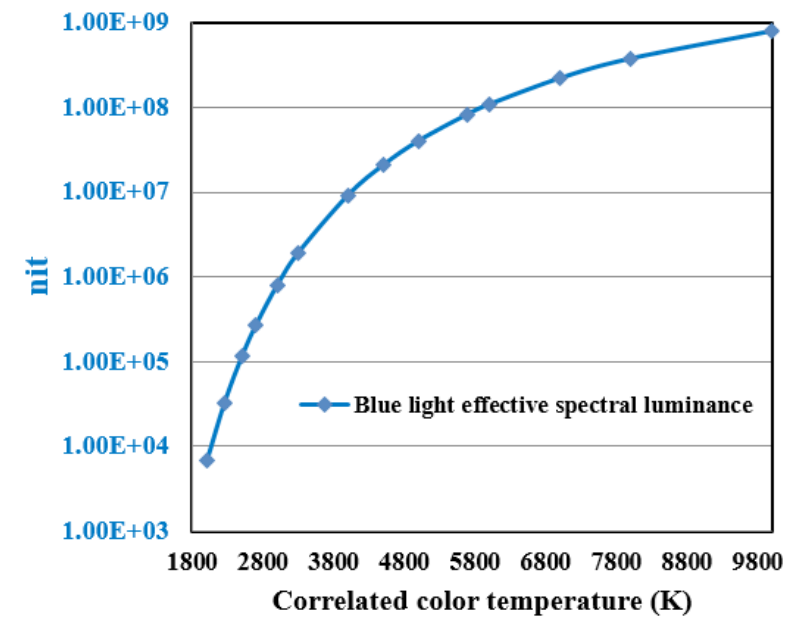

Our results are also in agreement with the work of Schulmeister et al., who conducted a study to evaluate the UV and blue light hazard of lamps based on some illuminance measurements and using a defined transformation factor referred to as the U-factor. U-factor can be achieved by dividing the blue light effective irradiance $\left(\mathrm{W} . \mathrm{m}^{-2}\right)$ by the illuminance level (lux) of the light source. The study revealed a good straight correlation between the U-factor and CCT of the lamps, and the reported amounts for the blue light effective irradiance corresponds well with our findings [14].

A strong correlation between $\mathrm{CCT}$ and a defined quantity called BLH efficacy of luminous radiation, $\mathrm{K}_{\mathrm{B}, \mathrm{v}}$, has also been expressed in International Electrotechnical Commission (IEC) 62778 standard. $\mathrm{K}_{\mathrm{B}, \mathrm{v}}$ with units in W.Im-1 is given by dividing the blue light effective radiance by the corresponding photometric quantity of luminance [24]. Higher $\mathrm{K}_{\mathrm{B}, \mathrm{v}}$ values are associated with higher CCTs, because higher CCTs lead to an increase in

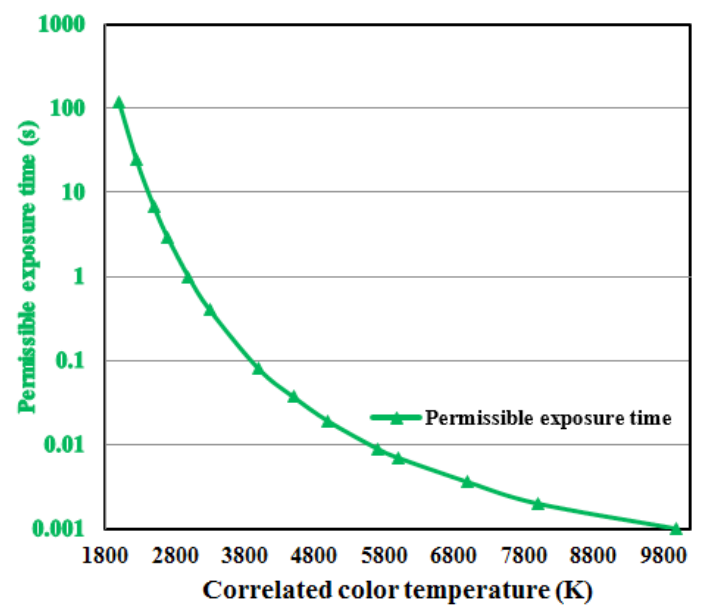

Figure 8. Estimated Effective Spectral Luminance (Left) and Estimated Permissible Exposure Duration (Right), Using Proposed Photometry Method for the CCT Range of 2000-10000 K 
the $\mathrm{K}_{\mathrm{B}, \mathrm{v}}$ numerator (blue light effective radiance), implying that LEDs with higher CCTs have greater blue light effective luminance.

For better understanding, the spectral distributions of LEDs with different CCTs are illustrated in Figure 6. For comparison, spectral weighting functions of $\mathrm{B}_{(\lambda)}, \mathrm{V}_{(\lambda)}$, and $\gamma_{(\lambda)}$ have been added. As Figure 6 shows, the LED light spectrum consists of two main peaks: a narrow blue and a wider yellow, and this is the case for all white LEDs regardless of CCT. The ratio of the intensity of one of these peaks to that of another determines whether the lamp looks cold or warm. As depicted in Figure 6, for lower CCTs $(2700-3700 \mathrm{~K})$, the relative proportion of the yellow part overcomes, and this leads to the higher effectiveness of $\mathrm{V}_{(\lambda)}$ compared to $\mathrm{B}_{(\lambda)}$. Therefore, for sources with lower CCTs, thanks to lower blue content, their blue light effective luminance decreased. These results are supported by Halbritter and associates [9].

Another reason that can be noticed as a support for our data is shown in Figure 7. The spectral distribution of LEDs having the same CCT produced by different lighting manufacturers lie well on each other, implying that a white LED with an assigned CCT will always emit light of a specified and estimable value of energy, blue light, and luminance. It then enables us to determine the amount of the proposed quantity "blue light effective luminance" of every white LED lamp with a certain CCT.

\section{Estimation of threshold exposure time using CCT of LED light sources}

After reliability analysis of the proposed photometry method, calculated values of blue light effective luminance, obtained from the conversion of blue light effective radiance data, can be employed to yield threshold exposure durations according to ICNIRP recommendations. Figure 8 shows the calculated blue light effective spectral luminance and the corresponding estimated permissible exposure durations using the proposed photometry method for the CCT range of 2000-10000 K. According to Figure 8, the permissible exposure time corresponding to the CCT range of $2000-10000 \mathrm{~K}$ was determined to fall within the 0.001-100 s range during a day, with higher exposure limits for lower CCTs, suggesting that LED light sources with higher CCT are more dangerous to the retina because sources with higher CCT tend to produce cooler light containing a higher ratio of blue light. Based on these findings, a maximum recommended CCT of $3000 \mathrm{~K}$ can be proposed to the lighting manufacturers and designers for optical radiation safety.
At the moment, most of the commercially available LED lamps fall within 2700-6500 K.

However, it can generally be said that four vital factors should be controlled to avoid retina injury: the spectral luminance distribution, blue light effective luminance intensity, exposure angle (the area of the retina exposed), and exposure duration. The risk relating to the former two is reduced through using lower CCT numbers, as previously mentioned. Designing the lighting system so that the light sources are not located directly in the field of view may be the solution for the latter two.

In this study, a mathematical equation was developed to determine a new proposed expression as "blue light effective luminance" using a new parameter of $\gamma_{(\lambda)}$ resulting from the multiplication of $\mathrm{B}_{(\lambda)}$ and $\mathrm{V}_{(\lambda)}$. Employing this equation (a computer code) and having knowledge of the CCT of LED lamps makes it possible to evaluate the blue light exposure limit without using any radiometer and unnecessary calculations for effective spectral radiance in cases where the measuring instrument (spectroradiometer) are not equipped with the $\mathrm{B}_{(\lambda)}$ filter [4].

It was described how the correlated color temperature of LED lamps in K could be substituted for the effective spectral radiance of the light source in $\mathrm{W} \cdot \mathrm{m}^{-2} \mathrm{sr}^{-1}$ to evaluate blue light photochemical exposure levels. We then explained that the approach could be extended to calculate the new term effective spectral luminance corresponding to "ICNIRP Guidelines on Limits of Exposure to Broad-band Optical Incoherent Radiation". The proposed method could estimate allowable exposure duration in any geometrical position where an observer views an LED lamp.

The results showed that in the proposed photometry method, the relative error resulting from excluding a narrow band of light $(300-380 \mathrm{~nm})$ varied from $0.03 \%$ to $0.78 \%$ for the CCT range of 2000 to $6000 \mathrm{~K}$, respectively. These values of relative approximate error are minor and acceptable for routine white LED light sources.

It can be concluded here that the proposed photometry method makes the concept of BLH very simple and more understandable. In addition to the fact that a measuring instrument cannot be available everywhere for BLH analysis, most of the threshold limit values related to non-ionizing radiation have been provided in the form of complex physical concepts and quantities which are difficult to understand by occupational hygienists, physicians, ophthalmologists, vision experts and other specialists dealing with photometry and non-ionizing 
radiation health and safety. The proposed photometry method allows us to determine the permissible exposure time by only knowing the CCT of the light source, and no additional calculations are required. An Originpro computing code can be conveniently employed for the computations to be carried out quickly.

\section{Conclusions}

Correlated color temperature is one of the main properties of every light source, including LED lamps. It is possible to use this photometric characteristic to estimate BLH without using light measuring devices. In the present study, the radiometric quantity (blue light effective radiance) in $\mathrm{W} \cdot \mathrm{m}^{-2} \mathrm{sr}^{-1}$ was converted to photometric quantity (blue light effective luminance) in nit, so that by using only the CCT of an LED lamp and applying a computer code, the exposure duration for any distance of the observer could be determined. The advantages of the proposed method are simplicity and the availability of color temperature of LED lamps. Still, currently for BLH estimation, radiometers equipped with blue light hazard function filter in the wavelength range of 300 to $700 \mathrm{~nm}$ are required. Most of the time color temperatures are specified by the lamp producers. However, when the color temperature is not provided, an LED color meter can be employed, which is more readily available.

\section{Ethical Considerations}

\section{Compliance with ethical guidelines}

There were no ethical considerations to be considered in this research.

\section{Funding}

The authors did not receive support from any organization for the submitted work.

\section{Authors' contributions}

All of the authors equally contributed to prepare this project.

\section{Conflict of interest}

The authors declared no conflict of interest.

\section{Acknowledgments}

The authors would like to thank Kurdistan University of Medical Sciences and Iran University of Medical Sciences for technical support and assistance.

\section{References}

[1] Behar-Cohen F, Martinsons C, Viénot F, Zissis G, BarlierSalsi A, Cesarini JP, et al. Light-Emitting Diodes (LED) for domestic lighting: Any risks for the eye? Prog Retin Eye Res. 2011; 30(4):239-57. [DOI:10.1016/j.preteyeres.2011.04.002] [PMID]

[2] Okuno T, Ojima J, Saito H. Blue-light hazard from $\mathrm{CO} 2$ arc welding of mild steel. Ann Occup Hyg. 2010; 54(3):293-8. [DOI:10.1093/annhyg/mep090] [PMID]

[3] Bullough JD, Mies MS. The blue-light hazard: A review. J Illum Eng Soc. 2000; 29(2):6-14. https:/ / www.researchgate. net/publication/286562716_The_blue-light_hazard_A_review

[4] Okuno T, Saito H, Ojima J. Evaluation of blue-light hazards from various light sources. Dev Ophthalmol. 2002; 35:104-12. [DOI:10.1159/000060814] [PMID]

[5] International Commission on Non-Ionizing Radiation Protection. ICNIRP statement on Light-Emitting Diodes (LEDs) and laser diodes: Implications for hazard assessment. Health Phys. 2000; 78(6):744-52. [DOI:10.1097/00004032-20000600000020] [PMID]

[6] Smith BT, Belani S, Ho AC. Ultraviolet and near-blue light effects on the eye. Int Ophthalmol Clin. 2005; 45(1):107-15. [DOI:10.1097/01.iio.0000148392.29642.3a] [PMID]

[7] Sliney DH. Optical radiation safety of medical light sources. Phys Med Biol. 1997; 42(5):981-96. [DOI:10.1088/00319155/42/5/016] [PMID]

[8] Sliney DH. Quantifying retinal irradiance levels in light damage experiments. Curr Eye Res. 1984; 3(1):175-9. [PMID]

[9] Halbritter W, Horak W, Jordan W. Simplified approach for classification the potential photobiological hazards of leds according to CIE S009. Light Eng. 2012; 20(1):113-20. https:// web.p.ebscohost.com/abstract?direct

[10] International Commission on Non-Ionizing Radiation Protection. ICNIRP guidelines on limits of exposure to incoherent visible and infrared radiation. Health Phys. 2013; 105(1):74-96. [DOI:10.1097/HP.0b013e318289a611]

[11] Michael R, Wegener A. Estimation of safe exposure time from an ophthalmic operating microscope with regard to ultraviolet radiation and blue-light hazards to the eye. J Opt Soc Am A Opt Image Sci Vis. 2004; 21(8):1388-92. [DOI:10.1364/ JOSAA.21.001388] [PMID]

[12] West KE, Jablonski MR, Warfield B, Cecil KS, James M, Ayers MA, et al. Blue light from light-emitting diodes elicits a dose-dependent suppression of melatonin in humans. J Appl Physiol (1985). 2011; 110(3):619-26. [DOI:10.1152/japplphysiol.01413.2009] [PMID]

[13] Weber M, Schulmeister K. Hazard assessment of lamps following the CIE lamp safety standard. https:/ / laser-led-lampsafety.seibersdorf-laboratories.at/

[14] Schulmeister K, Buberl A, Weber M, Kitz E. Simplified method to assess the UV and blue light hazard of lamps. InInternational Laser Safety Conference 2013 Mar (Vol. 2013, No. 1, pp. 357-365). Laser Institute of America. https://doi. org/10.2351/1.5056814 
[15] ACGIH. Light and Near-Infrared Radiation. In: TLV(R) and BEIs based on the documentation of the threshold limit values for chemical substances and physical agents \& biological exposure indics. Salt Lake City: Signature Publishing; 2015. http://dl.mozh.org/up/acgih-2015.pdf

[16] Meyer-Arendt JR. Radiometry and photometry: Units and conversion factors. Appl Opt. 1968; 7(10):2081-4. [DOI:10.1364/AO.7.002081] [PMID]

[17] McCluney R. Introduction to radiometry and photometry. Norwood: Artech House; 2014. https://books.google.com/ books?id=hnx-oAEACAAJ\&dq

[18] Madjidi F. Using temperature of IR sources for assessing photochemical and aphakic retinal hazard. Iran J Med Phys. 2016; 13(1):43-8. [DOI:10.22038/IJMP.2016.7144]

[19] Howell JR, Menguc MP, Siegel R. Thermal radiation heat transfer. $5^{\text {th }}$ ed. Boca Raton: CRC press; 2010. [DOI:10.1201/9781439894552]

[20] Planck M. The theory of heat radiation. New York: Dover Publications; 2013. https:// books.google.com/books?id=oN LCAgAAQBAJ\&printsec $=$ frontcover\&dq

[21] Krames MR, Shchekin OB, Mueller-Mach R, Mueller GO, Zhou L, Harbers G, et al. Status and future of high-power light-emitting diodes for solid-state lighting. J Disp Technol. 2007; 3(2):160-75. [DOI:10.1109/JDT.2007.895339]

[22] Davis R, Ginthner DN. Correlated color temperature, illuminance level, and the Kruithof curve. J Illum Eng Soc. 2013; 19(1):27-38. https://www.researchgate.net/publication/261571321_Correlated_Color_Temperature_Illuminance_Level_and_the_Kruithof_Curve

[23] Madjidi F, Behroozy A. Replacing effective spectral radiance by temperature in occupational exposure limits to protect against retinal thermal injury from light and near Ir radiation. J Occup Environ Hyg. 2014; 11(10):688-94. [PMID]

[24] IEC TR 62778. Application of IEC 62471 for the assessment of blue light hazard to light sources and luminaires [Internet]. 2014. https://webstore.iec.ch/publication/7427 